\title{
POPULATION STUDY ARTICLE Comparison of fetal growth by maternal prenatal acetaminophen use
}

\author{
Melissa M. Smarr $\mathbb{D}^{1,2}{ }^{1,}$ Joe Bible ${ }^{3}$, Nicole Gerlanc ${ }^{4}$, Germaine M. Buck Louis ${ }^{1,5}$, Alaina Bever ${ }^{1}$ and Katherine L. Grantz ${ }^{1}$
}

BACKGROUND: Equivocal findings exist regarding prenatal acetaminophen use and various adverse neonatal and childhood health outcomes, though with no data on fetal growth. We evaluated whether fetal growth differed by maternal acetaminophen use.

METHODS: Racially diverse, healthy women with low-risk antenatal profiles from 12 US clinical centers were enrolled in a prospective cohort study and followed until delivery. Ultrasound measurements of fetal parameters and self-reported prenatal acetaminophen use were collected at enrollment and up to five follow-up visits. Prenatal acetaminophen use was dichotomized as none or any.

RESULTS: Among 2291 women, 932 (41\%) reported the use of acetaminophen medications during the current pregnancy. Estimated growth curves of fetal parameters did not differ between women reporting use of any medication containing acetaminophen and women with no reported use of the same.

CONCLUSION: Among healthy mothers with low-risk pregnancies, maternal acetaminophen use was not associated with alterations in fetal growth.

Pediatric Research (2019) 86:261-268; https://doi.org/10.1038/s41390-019-0379-7

\section{INTRODUCTION}

The use of non-prescription medications is generally considered safe for non-pregnant adults, which could explain the increase in use of over-the-counter (OTC) drugs during pregnancy. ${ }^{1}$ Use of OTC medication occurs in an estimatWed $70 \%$ of pregnancies both nationally and internationally, ${ }^{2}$ and concerns about usage during pregnancy relative to fetal growth and development, in the absence of randomized clinical trials, remain. ${ }^{3}$ One commonly used OTC analgesic and antipyretic, acetaminophen, also known as paracetamol outside the United States and Canada, is found in a large number of OTC and prescription drugs. ${ }^{4}$ Maternal intake of acetaminophen during pregnancy is believed to be non-toxic in low doses and was previously listed as a Pregnancy Category B drug according to the US Food and Drug Administration. ${ }^{3}$ The drug of choice for pain relief among most pregnant women, prenatal acetaminophen use has been reported in national and international epidemiologic studies with frequencies ranging from 29.9 to $81 \% .^{5}$ Based on data from the National Birth Defect Prevention Study and the Boston University Slone Epidemiology Center Birth Defects Study, prevalence of acetaminophen use during any period of pregnancy is $65-70 \%$; the highest prevalence was reported in the first trimester (54.2\%), 50.5\% in the second trimester, and the lowest in the third trimester (48.0\%). ${ }^{1}$

Given the high prevalence of acetaminophen use during pregnancy and the ability of acetaminophen to freely cross the placenta, ${ }^{6,7}$ researchers are now focusing on the safety of maternal exposure to acetaminophen and its effects on fetal health. Short- and long-term consequences of acetaminophen use during pregnancy are inconsistent for both animal and human studies. ${ }^{8}$ Most of the previous literature is centered on acetaminophen use and observed birth defects in animal studies and increased odds of birth defects in human studies. In human studies, findings from a Danish prospective birth cohort study demonstrated that compared to mothers who reported no medication use, women who used paracetamol for more than 2 weeks during the first and second trimesters had increased risk of delivering boys with congenital cryptorchidism. ${ }^{9}$ Other European studies have reported similar findings with significant associations between maternal acetaminophen use and male reproductive disorders, highlighting second trimester as a potential critical window of exposure. ${ }^{10,11}$

Studies specific to fetal growth are limited and results are equivocal. Biologic plausibility stems from animal models, which have demonstrated that reduced fetal length in rat pups is associated with mid-gestation maternal exposure to 125 and 350 $\mathrm{mg}$ of acetaminophen, dosage comparable to maternal use. ${ }^{12,13}$ Various concentrations of acetaminophen have been associated with morphological changes in the fetal liver. It has been hypothesized that insults to the fetal liver may result in the reduction of blood stem cells to key organs, resulting in increased risk of adverse growth and development. ${ }^{12}$ Epidemiologic studies of prenatal acetaminophen use and fetal growth are non-existent. Our objective was to compare growth curves of fetal parameters between maternal prenatal acetaminophen use groups in a prospective cohort of low-risk, ethnically and geographically diverse maternal-fetal pairs.

\footnotetext{
${ }^{1}$ Division of Intramural Population Health Research, Eunice Kennedy Shriver National Institute of Child Health and Human Development, Bethesda, MD, USA; ${ }^{2}$ Department of Environmental Health, Rollins School of public Health, Emory University, Atlanta, GA, USA; ${ }^{3}$ School of Mathematical and Statistical Sciences, Clemson University, Clemson, SC, USA; ${ }^{4}$ The Prospective Group, Arlington, VA, USA and ${ }^{5}$ College of Health and Human Services, George Mason University, Fairfax, VA, USA Correspondence: Melissa M. Smarr (melissa.smarr@emory.edu)
} 


\section{PATIENTS AND METHODS}

Study sample

The NICHD Fetal Growth Study-Singletons was a multi-site, prospective cohort study designed to establish a standard for normal fetal growth (velocity) and size for gestational age for racially/ethnically diverse US pregnant women. The study cohort comprised 2334 non-obese (pregravid body mass index (BMI) $19.0-29.9 \mathrm{~kg} / \mathrm{m}^{2}$ ) gravidas who were recruited from 12 clinical sites over the course of 4 years (2009-2013) with representation from four self-identified race/ethnicity groups: non-Hispanic black ( $n=611,26 \%)$, Asian $(n=460,20 \%)$, non-Hispanic white $(n=614$, $26 \%)$, and Hispanic $(n=649,28 \%)$. Inclusion and exclusion criteria were specified to recruit healthy women with low-risk antenatal profiles consistent with optimal fetal growth. ${ }^{14}$ As such, women with major risk factors for altered fetal growth (hypertension, smoking, maternal vascular disease, etc.) were excluded from the study; full details of the study are provided elsewhere. ${ }^{15}$

Women were excluded from the current analysis if they did not meet the inclusion criteria after enrollment $(n=14,<1 \%)$, did not have ultrasound data available from at least one study exam $(n=$ $8,<1 \%)$, or did not report any data on medication use $(n=21,1 \%)$. Institutional review board approval was obtained from all participating sites; women gave informed consent before data collection.

Covariate data collection and self-reported acetaminophen use Women were interviewed about lifestyle, medical, and reproductive history upon enrollment and at each of the subsequent five prenatal visits. Details surrounding medication use during pregnancy was self-reported and abstracted from enrollment, study visit, and medical chart forms. During enrollment women were asked, "Since you became pregnant, have you used or taken any medications and non-prescription vitamins, minerals, herbals, and supplements?", and at each study visit, women were asked, "Since your last visit with us, have you used or taken any medications and non-prescription vitamins, minerals, herbals, and supplements?" with a response option of yes, no, refused, or don't know. For a yes response, participants could provide detailed information for each reported product including medication name (free text), whether it was prescription or non-prescription, route (i.e., by mouth, etc.), frequency, and duration of medication. At enrollment, women were asked "how often the medication was taken since pregnancy," and at each follow-up visit, women were asked "how often do/did you take the product?" (in addition to "refused" or "don't know," there were seven possible answers: "less than once a month," "once a month," "2-3 times a month (but less than once a week)," "1-2 times a week," "3-4 times a week," "5-6 times a week" and "everyday"). Additionally, women were asked whether they were still taking the reported medication at enrollment and during each follow-up visit. After delivery, abstraction of the medical charts included the name of every medication, including prescription, over the counter, and herbal medications, taken at any point during pregnancy along with the start date. Free-text maternal medication data was mapped to drug categories in the Slone Drug Dictionary with the use of the Coding Engine program. The dictionary and coding program have been used in multiple studies of medication use. ${ }^{16-18}$ The Slone Drug Dictionary listed 1177 acetaminophen-containing medications, of which a total of 24 unique medications were reported by women in our analytic cohort. Reported acetaminophen medications by active ingredients included primarily acetaminophen (Acetaminophen, Paracetamol, Tylenol, Tylenol Extra Strength, and Children's Tylenol); acetaminophen plus Diphenhydramine $\mathrm{HCl}$ (Tylenol PM); acetaminophen plus cough and/or nasal suppressants (Tylenol Cold, Tylenol Sinus, and Walgreens Cough Syrup); acetaminophen plus Aspirin (Excedrin and Excedrin Extra Strength); acetaminophen plus caffeine (Aspirin-Free Excedrin); acetaminophen plus hydrocodone (Lortab, Norco, and Vicodin); acetaminophen plus oxycodone (Tylox and Percocet); acetaminophen plus codeine (Tylenol with codeine and Tylenol \#3); acetaminophen plus butalbital (Fioricet and Phrenilin); acetaminophen plus propoxyphene (Darvocet); and acetaminophen plus isometheptene mucate, dichloralphenazone (Midrin).

\section{Assessment of fetal growth}

Each woman was randomized to one of four follow-up schedules to capture ultrasound data across the full range of gestation while minimizing ultrasound exposure. As such, five additional ultrasounds were scheduled for each woman within \pm 1 week of the targeted gestational age [baseline, visit $0: 8-13$ weeks; visit 1: 16-22 weeks; visit 2: 24-29 weeks; visit 3: 30-33 weeks; visit 4: 34-37 weeks; and visit 5: 38-41 weeks]. ${ }^{14}$ Fetal biometric measurements were performed at each visit according to standardized study procedures by study site sonographers who underwent credentialing that yielded excellent reliability for fetal measurements assessed via ultrasound examinations. ${ }^{19}$ Ultrasound examination included biparietal diameter (BPD, outer to inner), head circumference $(H C)$, abdominal circumference $(A C)$, and femur length (FL), all measured to the nearest $0.1 \mathrm{~mm}$. For all biometric measurements, three measurements were taken and averaged. Estimated fetal weight $(\mathrm{g})$ was calculated from $\mathrm{HC}, \mathrm{AC}$, and FL using the Hadlock formula. ${ }^{20} \mathrm{HC} / \mathrm{AC}$ ratio was calculated to assess symmetry of fetal growth. ${ }^{21} \mathrm{FL} / \mathrm{AC}$ ratio was calculated as an index to identify intrauterine growth-restricted fetuses by indirectly detecting the degree of subcutaneous fat and muscle loss, independent of gestational age. ${ }^{22}$ Study scans were captured using Voluson E8s (GE Healthcare, Milwaukee, WI, USA) with a transabdominal curved multifrequency volume transducer (realtime abdominal $4-8 \mathrm{MHz}$ ) and/or endovaginal multifrequency volume transducer (real-time intracavity $6-12 \mathrm{MHz}$ ). Study scans underwent a quality assurance (QA) process, during which $5 \%$ of study scans with triplicate images for each fetal measurement were randomly selected to be re-measured by the expert sonographer. ${ }^{23}$ The QA demonstrated excellent $(>0.90)$ reliability of biometric measurements between study sonographers and blinded expert reviewers.

\section{Statistical analysis}

Maternal gestational use pattern for acetaminophen-containing medications was dichotomized as no use or any use. These exposure categories were empirically selected given the potential for variable reporting of acetaminophen use. Maternal baseline characteristics were compared across gestational acetaminophen use groups using $x^{2}$ or Kruskal-Wallis tests for categorical or continuous data, respectively.

Longitudinal trajectories of each fetal parameter and EFW were estimated for each acetaminophen use group; curves were determined for the 10th, 50th, and 90th percentiles. Fetal biometric measurements and estimated fetal weight were logtransformed to normalize distributions. Using the Linear and Nonlinear Mixed Effects Models (nlme) R package, fetal parameter growth curves for each gestational acetaminophen use group were estimated from linear mixed models with cubic splines for gestational age with knots placed at the 25th, 50th, and 75th percentiles to evenly split the distributions. All mixed models included a random intercept and slope to account for the between- and within-subject variability for each mother-fetal pair. We performed likelihood ratio tests for global differences in curves for each fetal parameter. When the global test was significant ( $a=$ 0.05), we performed pairwise comparisons for gestational weekspecific differences of fetal parameter curves by categories of maternal acetaminophen use to assess significance using Wald $p$ values. Models were adjusted for maternal age, self-identified racial/ethnic group; type of insurance (private or managed care vs. other); education (less than high school, high school or equivalent, some college or associate degree, bachelor degree and master or 


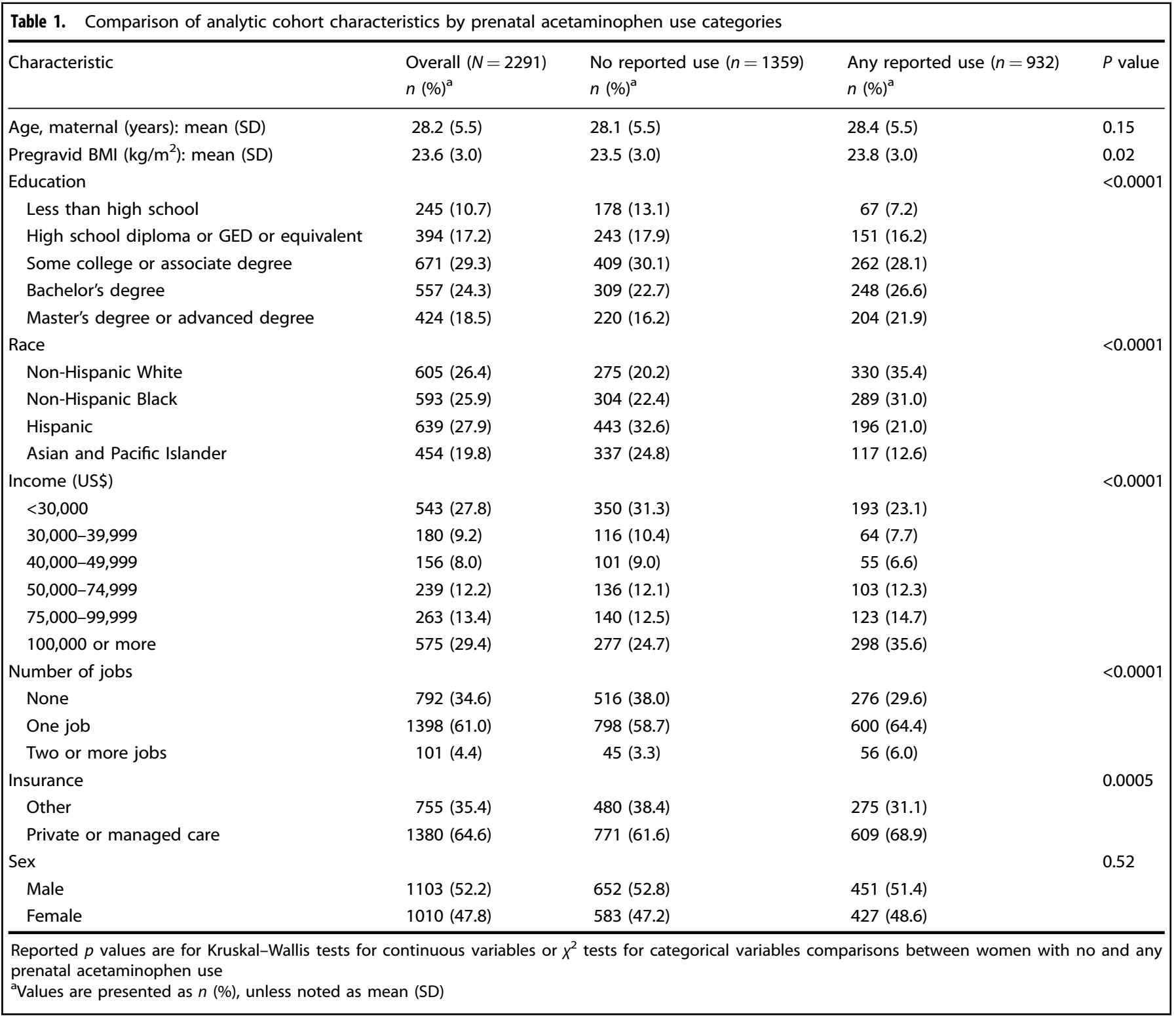

advanced degree); annual income ( $<$ US $\$ 30,000, \$ 30,000-39,999$, $\$ 40,000-49,999, \$ 50,000-74,999, \$ 75,000-99,999, \geq \$ 100,000)$; and the number of jobs worked (none, one, two, or more). The Multivariate Imputation by Chained Equations software for $\mathrm{R}$ was used to impute missing covariates for a total of 10 replications $(m=10)$ : highest level of education $(<1 \%)$, number of current paid jobs $(<1 \%)$, type of insurance $(8 \%)$, and income during last year (15\%), when performing adjusted tests for week-specific differences in fetal growth curves. Analyses were performed using SAS (version 9.4; SAS Institute Inc., Cary, NC, USA) or R (version 3.3.0).

\section{RESULTS}

Sample characteristics

Among the 2291 women (98\%) included in the analysis, 932 (41\%) reported the use of any medication containing acetaminophen during gestation. Of the prenatal acetaminophen users, 795 (85\%) reported only using medications with acetaminophen as the sole active ingredient throughout follow-up. Another 89 women (9\%), reported use of both medications with acetaminophen as the sole active ingredient and medications with other active ingredients (e.g., narcotics, antihistamines, nasal decongestants). An additional 54 women (6\%) reported only using medications with a primary active ingredient other than acetaminophen (e.g., narcotics, antihistamines, nasal decongestants) but also containing acetaminophen.

The average maternal age at study enrollment was $28.2 \pm 5.5$ years of age, and women generally had normal pregravid BMIs, $23.6 \pm 3.0 \mathrm{~kg} / \mathrm{m}^{2}$ as per study design (Table 1). Compared with women who reported no acetaminophen use during their pregnancy, reported usage of acetaminophen was more prevalent among non-Hispanic White and non-Hispanic Black women $(p<$ $0.0001)$. Additionally, having a college degree, an annual income $\geq \$ 100,000$, having one or more jobs, and having private insurance or managed care was more prevalent among women with any reported acetaminophen use, compared with those reporting no use ( $p$ values $\leq 0.001$, Table 1 ).

Estimates of change in growth of fetal biometry

Figure 1 presents the estimated curves of measured fetal parameters, BPD, HC, AC, and $\mathrm{FL}$, by gestational acetaminophen use category, including the median (50th), 5th, and 95th 

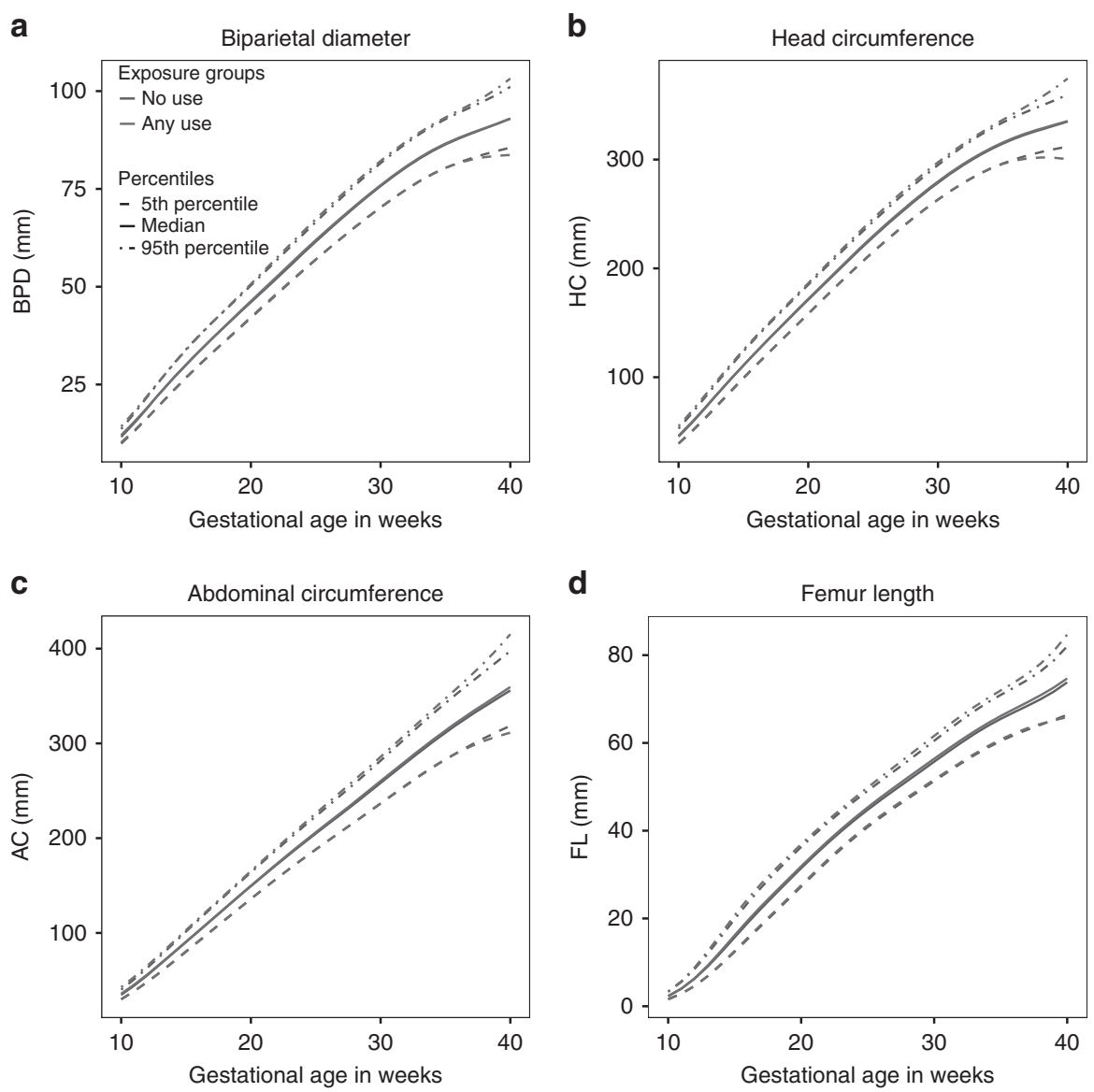

Fig. 1 Trajectories for measured fetal parameters by maternal acetaminophen use categories, NICHD Fetal Growth Studies-Singletons. Estimated 5th, 50th, and 95th percentiles for fetal biparietal diameter (a), head circumference (b), abdominal circumference (c), and femur length (d) by maternal self-reported prenatal acetaminophen use categories, as estimated from linear mixed models with log-transformed outcomes and cubic splines. mm, millimeters

percentiles. We found no significant differences in estimated fetal parameters across gestation between reported acetaminophen use groups (all gestational week-specific global $p$ values $\geq 0.05$ ). Estimated differences in fetal parameter means presented in Table 2 support the lack of variation between fetal growth parameters by reported prenatal acetaminophen use (pairwise comparison $p$ values $\geq 0.05$ ).

The fetal curves (median, 5th, and 95th percentiles) for estimated fetal parameters, EFW, HC/AC, and FL/AC, by acetaminophen use category are displayed in Fig. 2. Acetaminophen use patterns were not associated with differences in fetal EFW, HC/AC, and FL/AC (gestational week-specific global $p>0.05$ for all).

\section{DISCUSSION}

In a prospective cohort of healthy pregnant women with low-risk antenatal profiles, prenatal acetaminophen use was not associated with fetal growth. These findings are reassuring given that use was common (41\%) in our study population. Still, we exercise caution in our finding in light of our reliance on a rather global measure of self-reported usage that may not be sensitive to specific patterns of use. For example, most $(n=495 ; 53 \%)$ women who reported using any acetaminophen medications noted intermittent use, which may be associated with measurement error or bias.

It is difficult to compare our findings with previous epidemiologic studies, given the few population-based longitudinal studies currently addressing this question. Several earlier studies explored maternal use of acetaminophen during pregnancy and risk of allergic disorders (i.e., wheezing, asthma, eczema) in childhood $^{24-26}$ or neurodevelopment or behavioral outcomes (i.e., attention deficit hyperkinetic disorder). ${ }^{27,28}$ While some studies have reported increased odds ratio for amniotic band syndrome, with first trimester therapeutic use, ${ }^{29}$ to our knowledge none has examined the relationship between prenatal acetaminophen use and growth of core fetal biometrics, estimated fetal weight or parameter ratios indicative of asymmetric fetal growth.

\section{Strengths and limitations}

The NICHD Fetal Growth Studies was an ambitious undertaking to define new fetal growth standards for racially/ethnically diverse pregnant women in light of marked demographic changes in contemporary pregnant women. The major strength of our study was the prospective collection of both medication use and fetal growth measurements at multiple study visits across gestation. Furthermore, women in the NICHD Fetal Growth Studies overwhelmingly reported free text medication data at every study visit. Moreover, the classification of such medication data according to the reputable Slone Drug Dictionary and "Coding Engine" program, which identify medications according to a specific component, product, or group of products, limits potential exposure misclassification.

Potential limitations of this novel assessment include limited external validity or generalizability as our cohort, by design, was restricted to healthy women with low-risk antenatal profiles. This may, in part, be reflected in the large percentage of women (59\%) never reporting any prenatal acetaminophen use. We also recognize the potential limitations of assessing acetaminophen 
Comparison of fetal growth by maternal prenatal acetaminophen use MM Smarr et al.

Table 2. Fetal parameter differences and pairwise $p$ values between maternal prenatal acetaminophen use by gestational weeks, NICHD Fetal Growth Studies

\begin{tabular}{|c|c|c|c|}
\hline Fetal parameter & $\begin{array}{l}\text { Gestational age } \\
\text { (weeks) }\end{array}$ & $\begin{array}{l}\text { Difference: use } \\
\text { vs. no use }\end{array}$ & $P$ value ${ }^{b}$ \\
\hline \multirow{31}{*}{$\begin{array}{l}\text { Abdominal } \\
\text { circumference }(\mathrm{mm})\end{array}$} & 10 & 1.2 & 0.05 \\
\hline & 11 & 1.05 & 0.05 \\
\hline & 12 & 0.86 & 0.11 \\
\hline & 13 & 0.65 & 0.26 \\
\hline & 14 & 0.47 & 0.46 \\
\hline & 15 & 0.35 & 0.63 \\
\hline & 16 & 0.3 & 0.76 \\
\hline & 17 & 0.32 & 0.81 \\
\hline & 18 & 0.39 & 0.81 \\
\hline & 19 & 0.49 & 0.76 \\
\hline & 20 & 0.61 & 0.7 \\
\hline & 21 & 0.74 & 0.64 \\
\hline & 22 & 0.88 & 0.58 \\
\hline & 23 & 1.03 & 0.53 \\
\hline & 24 & 1.18 & 0.48 \\
\hline & 25 & 1.33 & 0.43 \\
\hline & 26 & 1.48 & 0.4 \\
\hline & 27 & 1.63 & 0.37 \\
\hline & 28 & 1.76 & 0.35 \\
\hline & 29 & 1.88 & 0.35 \\
\hline & 30 & 1.98 & 0.36 \\
\hline & 31 & 2.07 & 0.38 \\
\hline & 32 & 2.14 & 0.4 \\
\hline & 33 & 2.2 & 0.43 \\
\hline & 34 & 2.25 & 0.44 \\
\hline & 35 & 2.3 & 0.44 \\
\hline & 36 & 2.37 & 0.44 \\
\hline & 37 & 2.5 & 0.45 \\
\hline & 38 & 2.73 & 0.47 \\
\hline & 39 & 3.11 & 0.53 \\
\hline & 40 & 3.67 & 0.68 \\
\hline \multirow[t]{27}{*}{ Biparietal diameter (mm) } & 10 & 0.47 & 0.63 \\
\hline & 11 & 0.32 & 0.97 \\
\hline & 12 & 0.15 & 0.54 \\
\hline & 13 & 0 & 0.31 \\
\hline & 14 & -0.1 & 0.21 \\
\hline & 15 & -0.13 & 0.19 \\
\hline & 16 & -0.1 & 0.21 \\
\hline & 17 & -0.01 & 0.29 \\
\hline & 18 & 0.1 & 0.45 \\
\hline & 19 & 0.21 & 0.68 \\
\hline & 20 & 0.29 & 0.85 \\
\hline & 21 & 0.35 & 0.94 \\
\hline & 22 & 0.39 & 0.95 \\
\hline & 23 & 0.41 & 0.9 \\
\hline & 24 & 0.4 & 0.81 \\
\hline & 25 & 0.38 & 0.7 \\
\hline & 26 & 0.35 & 0.6 \\
\hline & 27 & 0.31 & 0.51 \\
\hline & 28 & 0.27 & 0.46 \\
\hline & 29 & 0.25 & 0.44 \\
\hline & 30 & 0.23 & 0.43 \\
\hline & 31 & 0.23 & 0.45 \\
\hline & 32 & 0.24 & 0.46 \\
\hline & 33 & 0.24 & 0.45 \\
\hline & 34 & 0.24 & 0.41 \\
\hline & 35 & 0.22 & 0.35 \\
\hline & 36 & 0.19 & 0.29 \\
\hline
\end{tabular}

\begin{tabular}{|c|c|c|c|}
\hline Fetal parameter & $\begin{array}{l}\text { Gestational age } \\
\text { (weeks) }\end{array}$ & $\begin{array}{l}\text { Difference: use } \\
\text { vs. no use }\end{array}$ & $P$ value $^{\mathrm{b}}$ \\
\hline \multirow{34}{*}{ Femur length $(\mathrm{mm})$} & $\begin{array}{l}37 \\
38\end{array}$ & $\begin{array}{l}0.14 \\
0.09\end{array}$ & $\begin{array}{l}0.25 \\
0.24\end{array}$ \\
\hline & 39 & 0.02 & 0.28 \\
\hline & 40 & -0.06 & 0.45 \\
\hline & 10 & -0.05 & 0.28 \\
\hline & 11 & 0.01 & 0.99 \\
\hline & 12 & 0.11 & 0.22 \\
\hline & 13 & 0.24 & 0.08 \\
\hline & 14 & 0.37 & 0.09 \\
\hline & 15 & 0.47 & 0.19 \\
\hline & 16 & 0.52 & 0.45 \\
\hline & 17 & 0.52 & 0.81 \\
\hline & 18 & 0.49 & 0.98 \\
\hline & 19 & 0.45 & 0.99 \\
\hline & 20 & 0.43 & 0.93 \\
\hline & 21 & 0.43 & 0.86 \\
\hline & 22 & 0.44 & 0.79 \\
\hline & 23 & 0.47 & 0.73 \\
\hline & 24 & 0.51 & 0.68 \\
\hline & 25 & 0.56 & 0.65 \\
\hline & 26 & 0.61 & 0.65 \\
\hline & 27 & 0.65 & 0.68 \\
\hline & 28 & 0.69 & 0.75 \\
\hline & 29 & 0.7 & 0.86 \\
\hline & 30 & 0.68 & 0.98 \\
\hline & 31 & 0.65 & 0.84 \\
\hline & 32 & 0.62 & 0.74 \\
\hline & 33 & 0.6 & 0.71 \\
\hline & 34 & 0.61 & 0.78 \\
\hline & 35 & 0.66 & 0.98 \\
\hline & 36 & 0.74 & 0.81 \\
\hline & 37 & 0.83 & 0.75 \\
\hline & 38 & 0.9 & 0.97 \\
\hline & 39 & 0.94 & 0.45 \\
\hline & 40 & 0.89 & 0.11 \\
\hline \multirow{26}{*}{ Head circumference(mm) } & 10 & 0.8 & 0.38 \\
\hline & 11 & 0.68 & 0.54 \\
\hline & 12 & 0.53 & 0.74 \\
\hline & 13 & 0.4 & 0.84 \\
\hline & 14 & 0.31 & 0.83 \\
\hline & 15 & 0.28 & 0.75 \\
\hline & 16 & 0.31 & 0.64 \\
\hline & 17 & 0.39 & 0.57 \\
\hline & 18 & 0.51 & 0.59 \\
\hline & 19 & 0.63 & 0.69 \\
\hline & 20 & 0.75 & 0.76 \\
\hline & 21 & 0.86 & 0.81 \\
\hline & 22 & 0.95 & 0.83 \\
\hline & 23 & 1.04 & 0.83 \\
\hline & 24 & 1.1 & 0.82 \\
\hline & 25 & 1.15 & 0.79 \\
\hline & 26 & 1.18 & 0.75 \\
\hline & 27 & 1.19 & 0.71 \\
\hline & 28 & 1.18 & 0.67 \\
\hline & 29 & 1.16 & 0.63 \\
\hline & 30 & 1.12 & 0.59 \\
\hline & 31 & 1.07 & 0.56 \\
\hline & 32 & 1.03 & 0.53 \\
\hline & 33 & 0.99 & 0.51 \\
\hline & 34 & 0.96 & 0.49 \\
\hline & 35 & 0.96 & 0.48 \\
\hline
\end{tabular}




\begin{tabular}{|c|c|c|c|}
\hline Fetal parameter & $\begin{array}{l}\text { Gestational age } \\
\text { (weeks) }\end{array}$ & $\begin{array}{l}\text { Difference: use } \\
\text { vs. no use }\end{array}$ & $P$ value ${ }^{b}$ \\
\hline \multirow{35}{*}{$\begin{array}{l}\text { Estimated fetal } \\
\text { weight }(g)\end{array}$} & $\begin{array}{l}36 \\
37\end{array}$ & $\begin{array}{l}0.96 \\
0.95\end{array}$ & $\begin{array}{l}0.49 \\
0.5\end{array}$ \\
\hline & 38 & 0.88 & 0.5 \\
\hline & 39 & 0.72 & 0.5 \\
\hline & 40 & 0.45 & 0.55 \\
\hline & 10 & 0.46 & 0.54 \\
\hline & 11 & 0.67 & 0.63 \\
\hline & 12 & 0.92 & 0.74 \\
\hline & 13 & 1.20 & 0.8 \\
\hline & 14 & 1.54 & 0.83 \\
\hline & 15 & 1.93 & 0.82 \\
\hline & 16 & 2.39 & 0.79 \\
\hline & 17 & 2.96 & 0.77 \\
\hline & 18 & 3.68 & 0.77 \\
\hline & 19 & 4.64 & 0.78 \\
\hline & 20 & 5.91 & 0.76 \\
\hline & 21 & 7.55 & 0.7 \\
\hline & 22 & 9.61 & 0.62 \\
\hline & 23 & 12.14 & 0.54 \\
\hline & 24 & 15.13 & 0.46 \\
\hline & 25 & 18.59 & 0.39 \\
\hline & 26 & 22.44 & 0.33 \\
\hline & 27 & 26.57 & 0.3 \\
\hline & 28 & 30.81 & 0.29 \\
\hline & 29 & 34.90 & 0.3 \\
\hline & 30 & 38.70 & 0.34 \\
\hline & 31 & 42.27 & 0.4 \\
\hline & 32 & 45.77 & 0.47 \\
\hline & 33 & 49.49 & 0.54 \\
\hline & 34 & 53.89 & 0.57 \\
\hline & 35 & 59.50 & 0.58 \\
\hline & 36 & 66.07 & 0.55 \\
\hline & 37 & 72.63 & 0.53 \\
\hline & 38 & 77.88 & 0.54 \\
\hline & 39 & 80.24 & 0.6 \\
\hline & 40 & 77.83 & 0.75 \\
\hline \multirow[t]{25}{*}{$\mathrm{HC} / \mathrm{AC}$} & 10 & -0.01 & 0.11 \\
\hline & 11 & -0.01 & 0.44 \\
\hline & 12 & -0.01 & 0.77 \\
\hline & 13 & -0.01 & 0.33 \\
\hline & 14 & 0.00 & 0.27 \\
\hline & 15 & 0.00 & 0.36 \\
\hline & 16 & 0.00 & 0.55 \\
\hline & 17 & 0.00 & 0.8 \\
\hline & 18 & 0.00 & 0.93 \\
\hline & 19 & 0.00 & 0.91 \\
\hline & 20 & 0.00 & 0.89 \\
\hline & 21 & 0.00 & 0.88 \\
\hline & 22 & 0.00 & 0.89 \\
\hline & 23 & 0.00 & 0.92 \\
\hline & 24 & 0.00 & 0.97 \\
\hline & 25 & 0.00 & 0.98 \\
\hline & 26 & 0.00 & 0.91 \\
\hline & 27 & 0.00 & 0.84 \\
\hline & 28 & 0.00 & 0.76 \\
\hline & 29 & 0.00 & 0.67 \\
\hline & 30 & 0.00 & 0.59 \\
\hline & 31 & 0.00 & 0.54 \\
\hline & 32 & 0.00 & 0.52 \\
\hline & 33 & 0.00 & 0.53 \\
\hline & 34 & 0.00 & 0.58 \\
\hline
\end{tabular}

\begin{tabular}{|c|c|c|c|}
\hline Fetal parameter & $\begin{array}{l}\text { Gestational age } \\
\text { (weeks) }\end{array}$ & $\begin{array}{l}\text { Difference: use } \\
\text { vs. no use }\end{array}$ & $P$ value $^{\mathrm{b}}$ \\
\hline \multirow{36}{*}{ FL/AC } & $\begin{array}{l}35 \\
36\end{array}$ & $\begin{array}{l}0.00 \\
0.00\end{array}$ & $\begin{array}{l}0.7 \\
0.82\end{array}$ \\
\hline & 37 & 0.00 & 0.87 \\
\hline & 38 & 0.00 & 0.78 \\
\hline & 39 & -0.01 & 0.51 \\
\hline & 40 & -0.01 & 0.3 \\
\hline & 10 & 0.00 & 0.06 \\
\hline & 11 & 0.00 & 0.03 \\
\hline & 12 & 0.00 & 0.05 \\
\hline & 13 & 0.00 & 0.1 \\
\hline & 14 & 0.00 & 0.19 \\
\hline & 15 & 0.00 & 0.27 \\
\hline & 16 & 0.00 & 0.33 \\
\hline & 17 & 0.00 & 0.39 \\
\hline & 18 & 0.00 & 0.45 \\
\hline & 19 & 0.00 & 0.5 \\
\hline & 20 & 0.00 & 0.51 \\
\hline & 21 & 0.00 & 0.48 \\
\hline & 22 & 0.00 & 0.42 \\
\hline & 23 & 0.00 & 0.34 \\
\hline & 24 & 0.00 & 0.27 \\
\hline & 25 & 0.00 & 0.2 \\
\hline & 26 & 0.00 & 0.16 \\
\hline & 27 & 0.00 & 0.12 \\
\hline & 28 & 0.00 & 0.1 \\
\hline & 29 & 0.00 & 0.08 \\
\hline & 30 & 0.00 & 0.08 \\
\hline & 31 & 0.00 & 0.09 \\
\hline & 32 & 0.00 & 0.1 \\
\hline & 33 & 0.00 & 0.11 \\
\hline & 34 & 0.00 & 0.12 \\
\hline & 35 & 0.00 & 0.12 \\
\hline & 36 & 0.00 & 0.12 \\
\hline & 37 & 0.00 & 0.13 \\
\hline & 38 & 0.00 & 0.13 \\
\hline & 39 & 0.00 & 0.16 \\
\hline & 40 & 0.00 & 0.29 \\
\hline
\end{tabular}

$H C / A C$ head circumference to abdominal circumference ratio, $F L / A C$ femur length-to-abdominal circumference ratio

${ }^{a} P$ values obtained by Wald test with adjustment for maternal age, selfidentified racial/ethnic group; type of insurance (private or managed care vs. other); education (less than high school, high school or equivalent, some college or associate degree, bachelor degree and master or advanced degree); annual income $(<\$ 30,000, \$ 30,000-39,999$, $\$ 40,000-49,999, \$ 50,000-74,999, \$ 75,000-99,999, \geq \$ 100,000)$; and the number of jobs worked (none, one, two, or more)

use with an open-ended questionnaire supplemented by chart review. There is the potential for under-reporting due to differences in recall or recognition of medications containing acetaminophen. Women in our cohort reporting acetaminophen use were more educated and had higher incomes than women with reported use. It remains possible that such women more reliably reported exposure or not if they had greater recognition of acetaminophen in other medications and reported it correctly. The extent to which such differences or other reporting biases may be generated with the use of self-administered questionnaires remains to be established. Another potential limitation is the lack of information on dosage provided by women in our cohort precluding a more formal investigation of potential dose-response relationships. Furthermore, while data on 
a

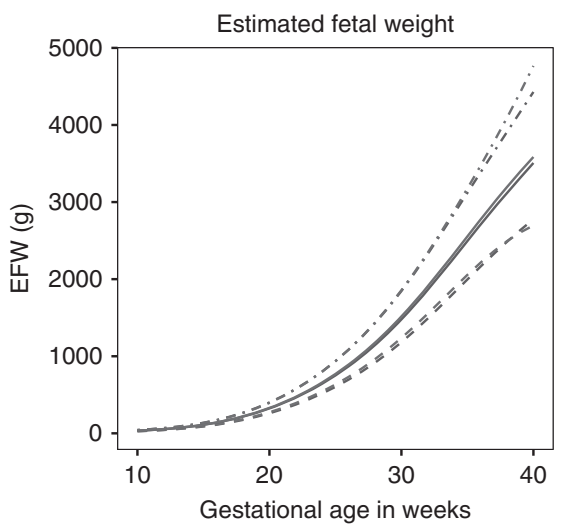

C

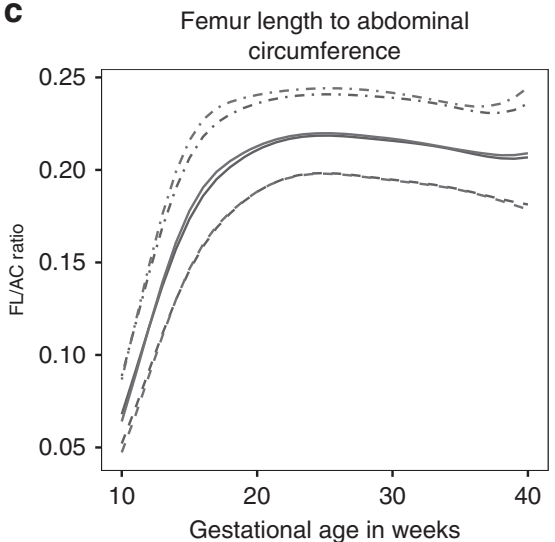

b

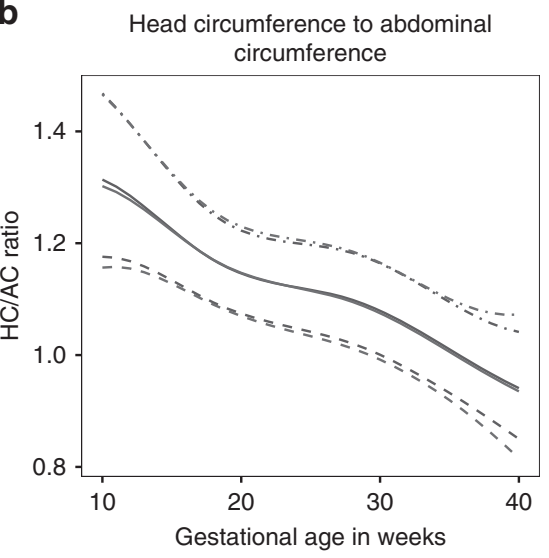

$$
\begin{aligned}
& \text { Exposure groups } \\
& \text { — No use } \\
& \text { — Any use } \\
& \text { Percentiles } \\
& \text { - } 5 \text { th Percentile } \\
& \text { - Median } \\
& \text { - . 95th Percentile }
\end{aligned}
$$

Fig. 2 Trajectories for derived fetal parameters by maternal acetaminophen use categories, NICHD Fetal Growth Studies-Singletons. Estimated 5th, 50th, and 95th percentiles for fetal estimated fetal weight (a), head circumference-to-abdominal circumference ratio (b), and femur length to abdominal circumference (c) by maternal self-reported prenatal acetaminophen use categories, as estimated from linear mixed models with log-transformed outcomes and cubic splines. g, grams

"frequency of medication use since pregnancy" was collected at enrollment and "frequency of use since last visit" was collected at each follow-up visit (Supplemental Table S1), the timing of followup is disjointed with the timing of exposure; meaning that we only know that exposure occurred sometime between the prior and current visits. The unknown timing (date of acetaminophen use) of exposure coupled with the sparse exposure patterns make modeling of growth trajectories as a function of temporally varying exposure (acetaminophen use) a non-trivial task. However, if we had coarsened the timing of use (i.e., trimesters), the exposure data would have resulted in sparse acetaminophen use patterns, which would have made the identification of distinct growth trajectories for each use profile unrealistic (Supplemental Table S2). Finally, we were unable to adjust for confounding by indication as not all women provided information on why they were taking medications containing acetaminophen.

\section{CONCLUSIONS}

Among healthy pregnant women, we found no evidence to support that maternal acetaminophen use was associated with altered fetal growth. With the recent attention placed on acetaminophen use and the potential for the Food and Drug Administration to re-evaluate the classification of medications containing this drug and their use during pregnancy, ${ }^{30}$ our findings may offer reassurance to mothers who have limited medication options and require pain or fever relief. Still, other important neonatal outcomes await purposeful investigation to ensure the absence of developmental toxicity.

\section{ACKNOWLEDGEMENTS}

We would like to acknowledge the research teams at all participating clinical centers, including Christina Care Health Systems; University of California, Irvine; Long Beach Memorial Medical Center; Northwestern University; Medical University of South Carolina; Columbia University; New York Hospital Queens; St. Peters' University Hospital; University of Alabama at Birmingham; Women and Infants Hospital of Rhode Island; Fountain Valley Regional Hospital and Medical Center; and Tufts University. Additionally, we acknowledge GE Healthcare Women's Health Ultrasound for their support and training on the Voluson and ViewPoint products over the course of this study, along with C-TASC and the EMMES Corporation for providing data and imaging support for this multi-site study. This work was supported by the Intramural Research Program of the Eunice Kennedy Shriver National Institute of Child Health and Human Development, contracts HHSN275200800013C; HHSN275200800002l; HHSN27500006; HHSN275200800003IC; HHSN275200800014C; HHSN275200800012C; HHSN275200800028C; HHSN275201000009C. The NICHD Fetal Growth Studies Clinical Trial Registration: ClinicalTrials.gov, NCT00912132.

\section{AUTHOR CONTRIBUTIONS}

All authors approved the final manuscript as submitted and agree to be accountable for all aspects of the work. M.M.S. conceptualized and performed the analysis, drafted the initial manuscript, and reviewed and revised the manuscript. J.B. oversaw the statistical analysis, and critically reviewed the manuscript. N.G. and MA.B. assisted with the imputation analysis, and critically reviewed the manuscript. G.M.B.L. and K.L.G. conceptualized and designed the study, and critically reviewed the manuscript.

\section{ADDITIONAL INFORMATION}

The online version of this article (https://doi.org/10.1038/s41390-019-0379-7) contains supplementary material, which is available to authorized users.

Competing interests: The authors declare no competing interests. 
Comparison of fetal growth by maternal prenatal acetaminophen use MM Smarr et al.

Publisher's note: Springer Nature remains neutral with regard to jurisdictional claims in published maps and institutional affiliations.

\section{REFERENCES}

1. Werler, M. M., Mitchell, A. A., Hernandez-Diaz, S. \& Honein, M. A. Use of over-thecounter medications during pregnancy. Am. J. Obstet. Gynecol. 193, 771-777 (2005).

2. Lupattelli, A. et al. Medication use in pregnancy: a cross-sectional, multinational web-based study. BMJ Open 4, e004365 (2014). https://doi.org/10.1136/bmjopen2013-004365

3. Servey, J. \& Chang, J. Over-the-counter medications in pregnancy. Am. Acad. Fam. Physicians 90, 548-555 (2014).

4. Lank, P. Acetaminophen. (American College of Medical Toxicology, 2014). https:// www.acmt.net/Acetaminophen.html Accessed: August 7, 2014.

5. Modick, H., Weiss, T., Dierkes, G., Bruning, T. \& Koch, H. M. Ubiquitous presence of paracetamol in human urine: sources and implications. Reproduction 147, R105-R117 (2014).

6. Byer, A. J., Traylor, T. R. \& Semmer, J. R. Acetaminophen overdose in the third trimester of pregnancy. JAMA 247, 3114-3115 (1982).

7. Peterson, A. M., \& Arcangelo, V. P. (eds) (2006). Pharmacotherapeutics for advanced practice: a practical approach. 2nd ed. Lippincott Williams \& Wilkins.

8. Thiele, K., Kessler, T., Arck, P., Erhardt, A. \& Tiegs, G. Acetaminophen and pregnancy: short- and long-term consequences for mother and child. J. Reprod. Immunol. 97, 128-139 (2013).

9. Kristensen, D. M. et al. Intrauterine exposure to mild analgesics is a risk factor for development of male reproductive disorders in human and rat. Hum. Reprod. 26, 235-244 (2011).

10. Jensen, M. S. et al. Maternal use of acetaminophen, ibuprofen, and acetylsalicylic acid during pregnancy and risk of cryptorchidism. Epidemiology 21, 779-785 (2010).

11. Philippat, C. et al. Analgesics during pregnancy and undescended testis. Epidemiology 22, 747-749 (2011)

12. Burdan, F., Siezieniewska, Z., Kis, G. \& Blicharski, T. Embryofetotoxicity of acetaminophen (paracetamol) in experimental in vivo model. Ann. Univ. Mariae Curie Sklodowska Med. 56, 89-94 (2001).

13. Lubawy, W. C. \& Garrett, R. J. Effects of aspirin and acetaminophen on fetal and placental growth in rats. J. Pharm. Sci. 66, 111-113 (1977).

14. Buck Louis, G. M. et al. Racial/ethnic standards for fetal growth: the NICHD Fetal Growth Studies. Am. J. Obstet. Gynecol. 213, 449.e1-449.e41 (2015).
15. Grewal, J. et al. Cohort Profile: NICHD Fetal Growth Studies-Singletons and Twins. Int. J. Epidemiol. 47, 25-25I (2018). https://doi.org/10.1093/ije/dyx161

16. Hall, S. A. et al. General exposures to prescription medications by race/ethnicity in a population-based sample: results from the Boston Area Community Health Survey. Pharmacoepidemiol. Drug. Saf. 19, 384-392 (2010).

17. Broussard, C. S. et al. Maternal treatment with opioid analgesics and risk for birth defects. Am. J. Obstet. Gynecol. 204, 314.e1-11 (2011).

18. Benedum, C. M., Yazdy, M. M., Mitchell, A. A. \& Werler, M. M. Impact of periconceptional use of nitrosatable drugs on the risk of neural tube defects. Am. J. Epidemiol. 182, 675-84 (2015).

19. American Institue of Ultrasound Medicine AIUM practice guideline for the performance of obstetric ultrasound examinations. J. Ultrasound Med. 259, 9 (2010).

20. Hadlock, F. P., Harrist, R. B., Sharman, R. S., Deter, R. L. \& Park, S. K. Estimation of fetal weight with the use of head, body, and femur measurements-a prospective study. Am. J. Obstet. Gynecol. 151, 333-337 (1985).

21. Peleg, D., Kennedy, C. M. \& Hunter, S. K. Intrauterine growth restriction: identification and management. Am. Fam. Physician 58, 453-460 (1998). 466-457.

22. Bamberg, C. \& Kalache, K. D. Prenatal diagnosis of fetal growth restriction. Semin. Fetal Neonatal Med. 9, 387-394 (2004).

23. Hediger, M. L. et al. Ultrasound quality assurance for singletons in the national institute of child health and human development fetal growth studies. J. Ultrasound Med. 35, 1725-1733 (2016).

24. Shaheen, S. O. et al. Prenatal paracetamol exposure and risk of asthma and elevated immunoglobulin E in childhood. Clin. Exp. Allergy 35, 18-25 (2005). S.

25. Persky, V. et al. Prenatal exposure to acetaminophen and respiratory symptoms in the first year of life. Ann. Allergy Asthma Immunol. 101, 271-278 (2008).

26. Garcia-Marcos, L., Sanchez-Solis, M. \& Perez-Fernandez, V. Early exposure to acetaminophen and allergic disorders. Curr. Opin. Allergy Clin. Immunol. 11, 162-173 (2011).

27. Liew, Z., Ritz, B., Rebordosa, C., Lee, P. C. \& Olsen, J. Acetaminophen use during pregnancy, behavioral problems, and hyperkinetic disorders. JAMA Pediatr. 168, 313-320 (2014).

28. Brandlistuen, R. E., Ystrom, E., Nulman, I., Koren, G. \& Nordeng, H. Prenatal paracetamol exposure and child neurodevelopment: a sibling-controlled cohort study. Int. J. Epidemiol. 42, 1702-1713 (2013).

29. Werler, M. M., Louik, C. \& Mitchell, A. A. Epidemiologic analysis of maternal factors and amniotic band defects. Birth Defects Res. A 67, 68-72 (2003).

30. FDA Drug Safety Communication: FDA has reviewed possible risks of pain medicine use during pregnancy [1-9-2015]. https://www.fda.gov/downloads/ Drugs/DrugSafety/UCM429119.pdf [Accessed, April 2015]. 